\title{
LA REFLEXION PREVENTIVE DE HABERMAS SUR LA TECHNIQUE GENETIQUE
}

Henri Mbulu*

RESUMO: O artigo procura reformar a argumentação de Habernas, a respeito do que ele denominou "engenismo liberal", ou seja, a possibilidade de os pais determinarem intervenções no genoma das células geminais fecundadas. $\mathrm{O}$ autor aprofunda a discussão ética sobre as consequêencias eugênicas de tais intervençốes apontadas por Habernas e revela a perplexidade do filósofo diante das insuficiências das respostas normativas a essa questão.

Jürgen Habermas est un penseur dont la réputation est telle qu'on ne le présente plus. Il est sans doute le plus important et le plus connu des philosophes allemands vivants. À 74 ans, il est professeur de philosophie et de sociologie à l'université Goethe de Francfort, en Allemagne. Héritier de l'École de Francfort et ancien assistant de Theodor Adorno, il conduit depuis plusieurs décennies une recherche sur la capacité de la raison à parvenir, par l'argumentation rationnelle dans l'espace public, à un consensus, c'est-à-dire à un accord entre sujets ou personnes concernées dans des actions communes.

* PhD, Chercheur au CRDP. Centre de recherche en droit public. Université de Montréal.
Dans son dernier ouvrage intitulé Die Zukunft der menschlichen Natur. Auf dem Weg zu einer liberalen Eugenik?,' traduit récemment en français, chez Gallimard, par Christian Bouchindhomme, sous le titre L'avenir de la nature humaine. Vers un eugénisme libéral?, ${ }^{2}$ Habermas nous livre sa réflexion préventive sur la technique génétique et ses conséquences eugéniques. Deux chapitres constituaient la publication originelle allemande. Ils ont été complétés par deux autres chapitres dans l'édition

\footnotetext{
1 Ouvrage publié chez Suhrkamp Verlag Frankfurt ani Main 2001.

2 Voir Jürgen HABERMAS, L'avenir de la nature humaine. Vers un éugénisme libéral? Gallimard, Paris, 2002 (ci-après: $A N H$ ). C'est à cette édition que nous nous référons.
} 
française. Le chapitre troisième est un Postscriptum à l'«eugénisme libéral». Il est un écho, outre-atlantique, des thèses de L'avenir de la nature humaine, dans le cadre d'un colloque, organisé à l'automne 2001, par Dworkin et Thomas Nagel, sur le thème Droit, philosophie et théorie sociale. ${ }^{3}$ Dans ce chapitre, Habermas répond aux objections des uns et des autres. Quant au chapitre quatrième, Foi et raison, il reprend une discussion qu' on croyait passé de mode, mais dont la rhétorique autour des événements du 11 septembre 2001, à New York, nous rappelle cruellement qu'elle est encore d'actualité. Toutefois, cette dernière discussion reste périphérique par rapport aux thèses entourant les effets pervers de la technique génétique.

Dans la présente communicatioen, il n'est ni possible ni même souhaitable de vouloir reprendre l'ensemble de l' argumentation que Habermas déploie dans l'ouvrage qui retient ici notre attention. Nous voulons simplement retracer la manière dont il conçoit son intervention dans le débat déclenché par la technique génétique. L'essentiel de ses thèses tourne autour de la question de l'«eugénisme libéral», expression traduisant le défi auquel nous confrontent les dérives de cette technique. Il entend par là «une pratique qui laisse à l'appréciation des parents la possibilité d'intervenir dans le génome des cellules germinales fécondées» $(A N H$, p. 117) de leur enfant à naître. Pour les fins de notre présentation, nous retiendrons deux thèses qui nous paraissent des plus significatives pour orienter notre réflexion vers le principe de précaution que Habermas mobilise. Nous y reviendrons plus en détail ultérieurement, cependant une énonciation préalable permettrait de les avoir présent à l'esprit.

Une première thèse découlant des incidences négatives des interventions génétiques peut se formuler comme suit: on ne saurait considérer comme moralement adéquat et légalement licite le diagnostic préimplantatoire à visée thérapeutique, sans glisser vers des manipulations génétiques semblables au «contrôle de qualité» (ANH, p. 51) des embryons. L'argument de la pente glissante, contenu dans cette thèse, a comme vocation de contrer les interventions à visée eugénique pratiquée par simple convenance.

Une deuxième thèse, plus fondamentale que la première, s'appuie sur l'affaissement de frontière que la technique génétique provoquerait dans la nature humaine entre le hasard naturel qui a gouverné jusqu'ici la procréation humaine et le libre choix relevant des interventions préférentielles dans le génome humain ( $A N H$, p. 48$)$. Cette thèse stipule que les interventions génétiques risquent de porter irrémédiablement préjudice aux conditions naturelles de la morale et du droit. Cela signifie en clair qu'on court-circuiterait une présupposition naturelle pour que une personne née puisse accéder à la conscience de pouvoir agir de manière libre, responsable et puisse se percevoir dans une égalité de naissance par rapport aux générations qui l'ont précédées. ${ }^{4}$ Il s'agit là, selon Habermas, d'un défi

4 Habermas explicite cette thèse au chapitre troisième, voir les pages 117 et svt., $A N H$. 
redoutable. Comment la philosophie s'en prend-t-elle à relever ce défi qui, pour l'instant n'est encore qu'hypothétique?

Dans la perspective de récents progrès des biotechnologies et des nouvelles possibilités d'intervention dans le génome humain, ${ }^{5}$ Habermas veut anticiper sur la discussion morale et non juridique. Il s'emploie à quitter la neutralité méthodologique de la philosophie dont l'éthique de la discussion s'est fait le modèle par excellence. Cette méthodologie lui permet d'opposer une éthique de la résistance - éthique de l'espèce humaine - à la technique génétique, en établissant, en filigrane, un principe de précaution. La crainte et la dangerosité morale que suscite cette technique sont fort probables. Car elle est devenue scientifiquement possible, médicalement utile et économiquement intéressante, à travers notamment les diagnostics prénatal et préimplantatoire ainsi que la recherche sur les embryons.

Pour mieux comprendre le sens de l'intervention et de deux thèses de Habermas, il n'est pas inutile de rappeler brièvement son parcours intellectuel et le contexte du débat auquel il prend part dans le cadre de nouvelles questions de bioéthique.

Par «parcours intellectuel», nous voulons simplement retracer les grandes orientations intellectuelles qui ont précédé la réflexion bioéthique de Habermas. Pour dire les choses

${ }^{5}$ Le séquençage du génome humain vient d'être achevé plus tôt que prévu. Salué par plusieurs médias d'information, le 20 avril 2003, il suscite, cependant, auprès de certains milieux des inquiétudes; non seulement par rapport aux droits d'auteur et aux désavantages que cela créerait pour les pays du TiersMonde, mais aussi sur les possibilités d'eugénisme qu'il recèle. rapidement, il est possible d'envisager trois différentes orientations ou périodes. La période de la philosophie théorique, pratique et appliquée. Les questions bioéthiques ou de l'éthique appliquée correspondent à la troisième période. Il serait simpliste de prétendre qu'il s'agit là d'un changement d'orientation qui délaisse les autres préoccupations, surtout pratique. Cependant, l'entrée remarquée, d'une grande figure de la philosophie morale et juridique contemporaine dans le domaine de la bioéthique, est plein de signification. Elle signifie que la philosophie pratique ne saurait plus ignorer les questions biopolitiques que soulèvent les développements technoscientifiques dans nos sociétés démocratiques. Elle démontre également que la philosophie pratique doit prendre ces questions au sérieux et ne saurait considérer la bioéthique ou l'éthique appliquée (quel que soit le nom qu'on lui donne) comme un service de sous-traitance de la culture philosophique; aucun objet n'est indigne de la philosophie.

Il reste que l'intérêt pour des problématiques bioéthiques est récent dans la pensée de Habermas. Ce dernier déclare aborder «ces questions sans familiarité originelle avec le champ de la bioéthique» (ANH, p. 169, note 13). Il est vrai que la préoccupation pour l'éthique appliquée est nouvelle dans l'ensemble de sa réflexion. Il ne s'est pas particulièrement intéressé à ce genre de questions dans lesquelles le philosophe siège parfois dans un cadre bureaucratique (de comité d'éthique ou autres), comme expert. Et comme expert, il est appelé à maîtriser plus l'art de la réglementation et de la décision que celui de la pensée réflexive. Mais comme intellectuel, il a un rôle social 
plus large à jouer. Le philosophe doit donc s'employer à plus de clarté dans les débats biopolitiques qui agitent les esprits de nos contemporains. Même si la discussion publique a toujours été une préoccupation majeure pour Habermas, il reste que son infléchissement vers des questions de la génétique est récent, comme le sujet lui-même.

On se rappellera que dans les années 1970, la pensée de Habermas s'intéressait beaucoup aux questions relatives à la philosophie théorique, en particulier à la pragmatique formelle et aux théories de la vérité. Depuis les années 1980, elle s'est tournée vers la philosophie pratique. L'essentiel de son œuvre, dans ces dernières années, sera consacré aux thèmes se rapportant au jugement moral et à l'agir dans l'univers postmétaphysique. Les multiples contextes socio-politiques de ces années ont, sans doute, été à la base de ce changement de cap. Il écrira plusieurs ouvrages durant cette période dont: Théorie de l'agir communicationnel en 1981; Morale et communication en 1983, Écrits politiques en 1985; De l'éthique de la discussion en 1991; Droit et démocratie. Entre faits et normes en 1992. Ce dernier livre est à la croisée de deux styles différents de réflexion. Il assure le passage de ses thèses en philosophie politique et morale vers la philosophie du droit.

Il faut constater qu'à ses thèses déjà fort bien connues en philosophie morale, politique, juridique et sur la démocratique délibérative, s'ajoute aujourd'hui, dans la réflexion de Habermas, un véritable essai de bioéthique. Il reste que cet essai est à prendre au sens littéral comme une tentative pour parvenir à un peu plus de transparence dans un écheveau d'intuitions quasiment inextricable. Les questions empiriques qu'il aborde sont d'une grande complexité et Habermas n'hésite pas à ajouter une note de modestie à ses analyses: «Je suis moi-même loin de croire être parvenu ne serait-ce qu'à la moitié de ce dessein. Mais je ne vois pas non plus d'analyses plus convaincantes» (ANH, p. 39).

Dans cet écheveau d'intuitions, constate Habermas, des questions de plusieurs ordres s'enchevêtrent. On peut nommer, de façon non exhaustive, celles de bioéthique soulevées par les nouvelles techniques de reproduction qui, depuis les années 1978, suscitent l'intérêt des médias et l'attention du public. La recherche sur les cellulessouches embryonnaires et sur des embryons surnuméraires n'a commencé que récemment vers les années 1998. Même si c'est depuis «1973 que des chercheurs sont parvenus à séparer et à recombiner des composants d'un génome» (ANH, p. 30), il reste que la technique génétique et ses applications en médecine de la procréation, telles les diagnostics prénatal et préimplantatoire, sont récentes. Dans la même foulée, le séquençage du génome humain a ensuite soulevé de grands espoirs de voir les thérapies géniques se développer à plus grande échelle. Ces espoirs s'accompagnent, sans doute, de l'intérêt économique pour leur exploitation par des compagnies pharmaceutiques. Sur un tout autre ordre, la querelle sur les progrès de la neurobiologie et les perspectives de manipulation des fonctions cérébrales, est aussi récente et suscite autant d'espoirs que d'inquiétudes. Car, l'on ignore la portée et les conséquences prévisibles que pourront 
avoir ces interventions génétiques sur l'espèce humaine.

Dans tous ces ordres de problèmes, il est évident qu'il ne s'agit encore que de conjectures. Pour l'heure, on ne saurait dire de façon indubitable ce qui relève de la «controverse à des bioscientifiques et à des ingénieurs exaltés par la science-fiction» ${ }^{6}$ de ce qui est de l'ordre d'une véritable prédiction scientifique. Mais prenant en compte le rythme souvent accéléré des divers progrès dans beaucoup de domaines de la vie, Habermas initie une réflexion préventive anticipant certaines éventualités en formulant, entre autres, les deux thèses déjà mentionnées pour s'opposer à la technique génétique. Il va sans dire qu'il n'est pas contre les interventions de la technique génétique en tant que telles, mais s'oppose au mode et à la portée de leur utilisation $(A N H$, p. 69). Cette opposition, Habermas l'inscrit dans la droite ligne de son parcours intellectuel. Néanmoins, il considère que les questions éthiques majeures qui se posent dans le contexte de la biogénétique requièrent une réponse d'une nature différente.

Il nous paraît important de situer, à présent, le contexte du débat allemand qui s'est déroulé, comme on peut l'imaginer dans un climat particulièrement tendu. À la différence d'autres pays occidentaux, comme, l'Angleterre, la France, l'Italie, etc., le débat allemand se déroulait sur fond d'un passé peu glorieux, d'une politique d'hygiène raciale eugénique qui recommandait, entre autres, l'euthanasie des handicapés. La race aryenne

\footnotetext{
6 ANH, p. 29.
}

n'était pas seulement «biologiquement» définie, mais aussi médicalement déterminée. Il ne suffisait pas d'être aryen. Il fallait être en bonne santé. Bref, l'hygiène raciale définissait les vies humaines qui valaient la peine d'être laissées en vie et celles qui n'en valaient point. On en tirait politiquement et socialement les conséquences. On comprend d'ailleurs, à partir de cet arrière-fond historique, pourquoi le débat sur l'avortement en Allemagne, le fameux article 218 du Code criminel, a fait couler beaucoup d'encre et provoqué plusieurs sursauts dramatiques. Après tout, l'embryon jouit d'une protection juridique. On comprendra surtout pourquoi les discussions portant sur une législation possible concernant le diagnostic préimplantatoire ou le diagnostic prénatal, ainsi que les recherches sur l'embryon ou les ćellules souches embryonnaires, ont ravivé des sentiments et des tensions plus vifs que dans d'autres pays. Tout se passait comme si les «vieux démons du nazisme» subitement se réveillaient de leurs cendres et annonçaient leur prochain retour légalement cautionné; d'autant plus que la Deutsche Forschungsgemeinschaft (DFG) l'équivalent d'un Conseil canadien de recherche ${ }^{7}$ - avait émis un avis favorable aux recherches sur les cellules souches embryonnaires, qui pouvait conduire à une révision plus libérale de la loi de 1990 sur la protection de l'embryon ( $A N H$, p. 32). La Deutsche Forschungsgemeinschaft proposait

\footnotetext{
7 Voir, en particulier, Lignes directrices des Instituts de recherche en santé du Canada, pour la recherche sur les cellules souches pluripotentes humaines, du lundi 4 mars 2002.
} 
d'accorder la priorité à la liberté de la recherche par rapport à la protection de l'embryon, car la recherche sur les débuts de la vie humaine ne consiste pas explicitement en la production d'embryons pour des fins de la recherche, mais de l'utilisation des embryons en vue de soigner. À l'instar de la Chambre des Lords au Royaume-Uni, ${ }^{8}$ la DFG soutenait que les chances d'utilisation des embryons à des fins thérapeutiques sont prometteuses ne serait-ce que par compassion pour nos congénères malades.

C'est dans ce contexte qu'il convient de placer l'intervention de Jürgen Habermas qui, partant du concept de l'éthique de l'espèce humaine s'interroge du bien fondé d'un tel avis à partir de sa réflexion philosophique antérieure mais surtout par référence aux prémisses de l'éthique de la discussion. ${ }^{9}$ Tout d'abord, nous aimerions ici reprendre aussi ses deux thèses précédemment énoncées avant d'aborder l'argument principal que nous voulons examiner, à savoir le principe habermasien de précaution, un concept qui se veut post-métaphysique. Résumons l'essentiel de la démarche argumentative.

\footnotetext{
${ }^{8}$ Au Royaume-Uni, la Human fertilisation and Embryology Act de 1990 définit les conditions dans lesquelles une recherche sur les embryons est autorisée. Une modification législative en 2002 permet la recherche sur les cellules souches embryonnaires et le clonage thérapeutique. Cependant, le clonage reproductif, comme dans la majorité des pays, est interdit.

9 Voir Jürgen HABERMAS, De l'éthique de la discussion, trad. M. Hunyadi, Paris, Cerf, 1992; Théorie de l'agir communicationnel, 2 tomes, trad. J.-M. Ferry et J.-L. Schlegel, Paris, Fayard, 1987; Morale et communication, trad. Chr. Bouchindhomme, Paris, Cerf, 1986.
}

Partant du plaidoyer pour un eugénisme ${ }^{10}$ libéral défendu par Buchanan, ${ }^{11}$ Brock et d'autres d'une part et, d'autre part du débat sur la recherche des embryons et des cellules souches qui a actuellement lieu au parlement allemand pour modifier la loi de 1990 sur la protection de l'embryon, ${ }^{12}$ Jürgen Habermas se garde bien de prendre directement position sans toutefois renvoyer dos à dos les positions qu'il appelle métaphysiques ou ontologiques concernant la dignité humaine. Quitte à revenir sur la portée de cette notion dans l'espace démocratique pluraliste moderne; nous aimerions exposer sa reformulation du problème.

De quelque sorte qu'elle soit, la technique génétique est une intervention dans le patrimoine génétique de l'homme dont il transforme la nature. Redoutable problème que Habermas traduit en termes d'instrumentalisation de la vie anténatale et d'atteinte à la dignité de la vie humaine.

Dans le débat allemand sur les questions du statut de l'embryon (diagnostics prénatal, préimplantatoire et la recherche sur les embryons); constate Habermas, «les participants au dialogue philosophique, en s'appuyant le plus souvent sur un concept de personne normativement saturé et sur une conception de la nature métaphysiquement chargée,

10 Voir sur le même thème de l'eugénisme: Catherine BACHELARD-JOBARD, L'eugénisme la science et le droit, Presse Universitaire de France, Paris, 2001.

1 Allen BUCHANAN, et al., From Chance to Choice. Genetics and Justice, Cambridge, Cambridge University Press, 2001.

12 En effet, la Embryonenschutzgesetz de 1990, en Allemagne, interdit toute recherche sur l'embryon qui n'est pas dans l'intérêt de l'embryon concerné. 
entrent dans le débat de principes qui vise à soumettre à un examen plutôt sceptique ce que les développements futurs de la technologie génétique [...] ont encore de conditionnel, en revanche ce qui intéressent les philosophes américains c'est principalement la manière dont ces développements vont trouver leur place, étant entendu qu'ils ne sont plus en principe remis en question et que, puisque l'application des thérapies géniques est un fait acquis, ils aboutiront à $\mathrm{du}$ shopping in the genetic supermarket. Certes, ces techniques interviendront dans le lien intergénérationnel en le bouleversant de fond en comble. Mais pour les philosophes américains, qui pensent pragmatiquement, les nouvelles pratiques ne soulèvent fondamentalement aucun nouveau problème; elles ne font que rendre plus aiguës les anciennes questions relatives à la justice distributive [...]» $(A N H$, p. 113).

On peut dégager ici deux principes clairs en faveur de la première thèse que défend Habermas. Premièrement, le débat allemand enferme la discussion actuelle sur la technique génétique dans une polarité semblable à celle qui a déjà eu lieu au sujet de l'avortement entre pro-vie et pro-choix. Ce débat est stérile et le rôle d'une réflexion philosophique est de nous sortir de la polarisation. C'est donc à partir des sobres prémisses postmétaphysiques de l'État de droit constitutionnel dans le cadre d'une société pluraliste, que Habermas voudrait apporter une contribution visant par la discussion à faire le point sur les sentiments moraux que les effets de la technique génétique réveillent. Ces prémisses considèrent, par exemple, qu'on ne saurait attribuer, à la vie prénatale, la dignité au sens éthique et juridique du terme. Toutefois cette vie mérite protection et le respect que nous accordons généralement à la vie humaine; d'où la distinction importante entre la dignité humaine et la dignité de la vie humaine.

Deuxièmement, la perspective de la recherche biogénétique, qu'envisagent les philosophes américains (Allen Buchanan, Thomas Nagel, Thomas McCarthy et d'autres) autour des questions de la justice distributive, ne va pas au fond des choses. Certes les inégalités sociales déjà existantes, aussi bien au niveau national.qu'international, se renforceraient si la technique génétique et son eugénisme libéral venaient à être «complètement victorieuses», ${ }^{13}$ pour reprendre l'expression d'Hannah Arendt. Mais cette position pragmatique américaine commet une erreur de jugement et une grave omission. D'un côté, elle se trompe en ne prenant pas au sérieux l'argument de la pente glissante derrière la thérapie génique $(A N H$, p. 35). De l'autre, elle omet gravement de s'interroger préalablement sur les conditions initiales de la liberté, de l'égalité et de la responsabilité. Pour Habermas, il en va tout autrement. Il stigmatise, dans sa première thèse, l'argument de la pente glissante. Et dans la deuxième, il s'intéresse, en particulier, aux conditions initiales de la morale et du droit; d'où la question: comment notre vision de nous-mêmes en tant que personnes, menant leur propre vie et ayant à rendre compte de leurs actes, se transformera-t-elle si nous en venons, un jour, à nous habituer à manipuler nos dispositions génétiques ou nos fonctions cérébrales?

13 Hannah ARENDT, L'Impérialisme, Fayard, Paris, 182, p. 291. 
Question sans doute redoutable, mais qui prend chez Habermas la forme d'une deuxième thèse qui, sans contredire l'énonciation que nous en avions donné, ${ }^{14}$ se précise comme de la manière suivante: «Je gage en effet que le fait de savoir que son génome personnel a été programmé est un facteur propre à perturber l'évidence en vertu de laquelle nous existons en tant que corps et «sommes», dans une certaine mesure, notre corps, et que de ce fait va naître un nouveau type de relation singulièrement asymétrique entre les personnes». ${ }^{15}$

Cette thèse vient confirmer l'hypothèse selon laquelle le grand danger, qu'engendrent les biogénétiques, contraint à une extrême prudence. Car les interventions dans l'équipement génétique humain risquent de bouleverser nos conceptions de la vie humaine antépersonnelle et la compréhension que nous avons de nous-mêmes comme étant éthiquement autonomes et soumis à une obligation sui generis de symétrie. Les interventions génétiques déstabilisent les conditions naturelles de la vie en introduisant une fracture au sein de la nature humaine. Alors que notre nature était jusque-là quelque chose de donné et d'intangible, elle est désormais susceptible de devenir objet de manipulations et de programmations, par lequel une personne interviendrait intentionnellement en fonction de ses préférences dans la base génétique et les dispositions naturelles d'autrui. En privant le titulaire du droit du «pouvoir-d'être-soimême» (ANH, pp. 82; 99), la recherche

\footnotetext{
14 Voir supra, p. 2.

${ }^{15}$ ANH, p. 68.
}

biogénétique et la programmation eugénique ouvrent la porte à la possibilité du bébé design et à la chosification de l'espèce humaine. Avec l'utilisation de la recherche biogénétique, nous atteignons donc les limites de l'invention humaine et l'impossibilité de disposer à notre guise des embryons; car il s'agit du domaine de l'indisponibilité de la nature humaine, dans lequel l'art, sauf pour des raisons thérapeutiques, ne semble plus bien produire ce que la nature n'a pu mener à bien, nouveauté que ne connaissait pas la pensée d'Aristote. Elle menace l'espèce humaine.

À l'égard de la menace d'un effacement de frontière entre la nature et l'invention ou l'art de la fabrication, entre les personnes et les choses risque également de se trouver remise en question, estime Habermas, la compréhension que nous avons de nousmêmes comme êtres normatifs, et par là même le fondement de la conscience morale, de la société démocratique et de l'humanité dans son ensemble. L'un des éléments de cette conscience est la certitude selon laquelle nous sommes capables de faire en sorte que nos opinions et nos attitudes ne soient déterminées que par des motifs raisonnables. Si cette conscience de la liberté était tacitement sapée par des pratiques normalisées de téléguidage, nos institutions démocratiques reposeraient sur des sables mouvants. Et sur des sables mouvants, comme le disait Niklas Luhmann, on ne peut rien bâtir de solide.

Pour étayer l'argument du préjudice eugénique qui découlerait des pratiques de téléguidage, Habermas imagine les allégations qu'un adolescent pourrait faire s'il apprend que son bagage génétique a été 
manipulé avant sa naissance, sans aucune raison thérapeutique contraignante, mais simplement selon les préférences de ses parents. L'adolescent pourrait ne pas s'identifier à cette dotation génétique. Par ailleurs, il se peut que les parents aient initié cette intervention eugénique avec les meilleures intentions d'optimiser les chances de réussite de la vie future de l'enfant à naître. Mais le fait qu'ils aient été aiguillés par leurs désirs arbitraires devient problématique. Car, il est loin d'être sûr que l'adolescent intègre les systèmes de valeurs et les préférences de ses parents. Dans cette perspective, il risque de remettre en cause le choix paternaliste de ses parents. Par exemple, il pourra se demander pourquoi ses parents l'ont doté d'un don mathématique plutôt que de capacités athlétiques ou musicales qui auraient été plus utiles pour la carrière de sportif de haut niveau ou de pianiste qu'il désire embrasser.

Il ne fait aucun doute pour Habermas que du seul fait que nous ne soyons pas à mesure d'exclure la possibilité d'un conflit entre adolescent programmé et parents, suffit amplement à prouver que le paternalisme eugénique est moralement condamnable. $\mathrm{Ce}$ paternalisme ne le prive certes pas de droits au sens juridique du terme, mais porte préjudice à sa conscience morale. Car le préjudice «consiste bien en ceci que l'adolescent concerné n'acquiert qu'une conscience précaire de son statut de titulaire des droits civiques. Il court le danger, au moment où il prend conscience de la contingence de son origine naturelle, d'être privé d'une condition mentale pour accéder à un statut sans lequel il ne peut accéder, en tant que sujet de droit, à la jouissance effective de l'égalité des droits» (ANH, p. 117).

Pour montrer le caractère irrécusable de ce préjudice, Habermas se fait une objection à lui-même: «Dire que les interventions eugéniques par transformation.génétique sont à même de transformer la structure d'ensemble de notre expérience morale est une affirmation forte» (ANH, p. 48). Mais il répond aussitôt à sa propre objection et à la question de savoir si nous voulons éroder la compréhension normative qu'ont d'ellesmêmes des personnes ayant le souci de mener leur vie et se portant mutuellement les uns aux autres un égal respect. L'humanité d'aujourd'hui doit faire un choix déterminant entre deux options. Ou bien, il faut choisir l'accroissement de la liberté, et donc le rejet de l'aliénation par les interventions génétiques; ou bien, il faut se faire complice, par la technique génétique, de la régression de l'autonomie et donc l'acceptation de saper les bases de la morale et du droit.

Partant de cette alternative, Habermas souligne que «si c'est la seconde éventualité qui se révèle exacte, le fait est que nous n'aurons pas dans l'immédiat d'argument moral concluant, mais une orientation inspirée par une éthique de l'espèce humaine, qui nous conseille la prudence et la modération» (ANH, p. 48-49). Dit autrement, l'éthique de l'espèce humaine conseille la retenue justifiée et nous incite à être prudents par rapport à la technique génétique. La prudence ne se situe pas dans la considération de l'eugénisme positif parce qu'il est mauvais en soi, mais dans la pente glissante que constitue l'eugénisme négatif dont on croît naïvement inoffensif. Il s'agit ici d'une formulation quelque peu modérée du 
principe de précaution en tant que réponse postmétaphysique à la question de la technique génétique.

Par ailleurs, cette formulation s'accompagne d'une autre plus incisive. Dans un passage qui ne saurait passer inaperçu, Habermas, s'opposant aux interventions eugéniques, ajoute: «lorsque des raisons morales contraignantes nous font défaut, il faut pour trouver notre chemin nous en tenir aux indications d'une éthique de l'espèce» ${ }^{16}$ (ANH, 107). Par cette injonction «il faut ... nous en tenir», il ne fait plus que conseiller la modération et la prudence. Il tente de tirer des conséquences de l'ensemble des interventions eugéniques et du glissement entre l'eugénisme négatif et l'eugénisme positif. Ce dernier rapport introduit une démarcation inacceptable. En effet, les frontières existant entre la thérapie génique et l'eugénisme de simple convenance, étant floues, Habermas se montre ici plus que réservé face à la technique génétique et aux manipulations sur le génome humain. Il précise: «dans la mesure où, pour des raisons conceptuelles et pratiques, cette ligne de démarcation est fluctuante, le préalable qui visait à contenir les interventions génétiques en deçà d'une frontière au-delà de laquelle on serait en présence d'améliorations des caractéristiques génétiques, nous confronte toutefois à un défi paradoxal puisque nous sommes amenés, dans un domaine précisément où les frontières sont fluctuantes; à établir et à imposer des lignes de démarcation particulièrement précises. C'est là l'argument qui, au demeurant, sert déjà aujourd'hui à défendre un eugénisme libéral qui ne connaît pas de frontière entre les interventions thérapeutiques et les interventions à des fins d'amélioration, mais laisse aux préférences individuelles des acteurs du marché le choix des finalités qui président aux interventions destinées à modifier les caractéristiques génétiques». ${ }^{17}$ Fort de cet argument, il en vient à prescrire une protection quasi absolue de la vie anténatale. Sans doute, avons-nous affaire ici à une formulation forte du principe de précaution.

Tout compte fait, ces deux formulations sont les critères du principe de précaution que Habermas établit pour protéger l'espèce humaine des dérives de la technique génétique, c'est-à-dire l'eugénisme libéral. La première formulation nous semble faible ou modérée alors que la seconde paraît plus radicale. De la sorte, le rapport entre ces deux formulations devient délicat à trancher. Toutefois, si notre lecture de deux thèses (pente glissante et préjudice) est juste, la deuxième formulation devrait logiquement annihiler la première. Car une éthique de l'espèce humaine ne saurait accepter de demimesure, «la manœuvre à demi convaincue de la DGF» (ANH, p. 33), ni prescrire une précaution qui ne protège pas suffisamment l'avenir de la nature humaine. C'est pourquoi, il faut poser des indications claires de l'éthique de l'espèce humaine qui interdisent l'eugénisme libéral, sous sa forme aussi bien positive que négative, et met ainsi l'espèce humaine à l'abri de la réification.

Malgré cette conclusion qui nous semble logique, les choses ne sont pas aussi tranchées

\footnotetext{
${ }^{16}$ Nous soulignons.
}

${ }^{17} A N H$, p. $34-35$ 
dans la pensée de Habermas; tout est en nuance. Mais c'est la prééminence de la version forte qui nous semble correspondre le plus avec une éthique dont la préoccupation majeure est la préservation de l'espèce humaine. Toujours est-il qu'on ne saurait nier que, face à l'eugénisme, en particulier, négatif, Habermas conseille, dans sa version faible, la prudence et la modération, c'est-àdire une retenue justifiée par les arguments postmétaphysiques de la «juste vie». Dès lors la question se pose de savoir si Habermas est vraiment contre (a) la thérapie génique et (b) toutes les interventions sur le génome humain. Loin de nous l'idée de lui faire passer un test de vérité, il s'agit simplement de constater la cohérence de ses thèses par rapport à son principe de précaution.

La réponse à la question (a) n'est pas simple. Certes Habermas n'ait pas naturellement contre les interventions génétiques justifiées par une nécessité thérapeutique. Mais, comme nous l'avons montré, les frontières entre la thérapie génique et l'eugénisme positif étant minces, il n'a point de cesse que de se montrer, au contraire, plus que réservé face aux manipulations eugéniques. Car en visant à soigner ou à prévenir des pathologies héréditaires, la thérapie génique contribue malgré tout à améliorer le patrimoine génétique humain et relève finalement de l'eugénisme au sens strict (ANH, p. 34-35). En vertu de l'argument de la pente glissante, Habermas est conduit à la protection absolue de l'embryon et à l'interdiction de la technique génétique, même s'il introduit une exception concernant les interventions thérapeutiques, c'est-à-dire les interventions curatives. Que vaut réellement cette exception sur le plan moral?
Quant à la question (b), Habermas n'est pas non plus contre toutes les interventions sur le génome humain, surtout celles à visée clinique préventive. On sait que la problématique qui l'intéresse était liée à la probabilité du danger que représente «le déplacement de la frontière entre hasard et libre choix» (ANH, p. 48) mais aussi le pouvoir-d'être-soi-même. Il se trouve que ce danger est inexistant lorsqu'une intervention sur le génome est destinée, non pas à changer les caractéristiques d'une personne à venir, mais à réaliser une finalité thérapeutique, c'est-à-dire pour le plus grand intérêt d'une personne dont on peut raisonnablement supputer le consentement. C'est le cas notamment des maladies héréditaires porteuses d'une tare congénitale. Pour les traiter ou les prévenir, elles doivent faire préalablement l'objet d'un diagnostic et d'un pronostic certains. Car, dans une société pluraliste, les finalités thérapeutiques sont les seuls cas dont on peut présumer, sans grand risque d'erreur, un large consensus social. C'est pourquoi Habermas pense qu'il appartiendra aux institutions démocratiques d'établir régulièrement une nomenclature des interventions jugées licites ou illicites, en mettant soigneusement en balance les avantages et les inconvénients de chaque pratique. Toute extension, abrogation ou amendement de la loi, représentant un risque grave ou sérieux, aussi bien pour les parents que pour l'enfant à naître, devrait être soumis au législateur. Les parents doivent être déchargés de la lourde responsabilité de négligence volontaire et ne doivent pas vivre dans la craindre du ressentiment que le futur enfant pourrait leur adresser. En introduisant, de nouveau, ces exceptions il en appelle à 
un haut besoin de réglementation des procédures eugéniques pour minimiser les risques éventuels.

Nous avons montré que placées dans le contexte de sociétés postséculières, les deux thèses du préjudice génétique et de la pente glissante des interventions eugéniques permettent à Habermas de mobiliser, contre l'eugénisme libéral, un double principe de précaution. Par contre, ce principe de l'éthique de l'espèce humaine, lorsqu'on le considère dans son sens fort, soulève de sérieuses questions de cohérence normative par rapport aux sobres prémisses d'un État démocratique.

Malgré tout, il faut conclure qu'en une période où il est de bon ton de saluer, par les médias et le grand public, les prouesses de la technique génétique, on peut voir dans la réflexion préventive de Habermas et ses mises en garde contre l'eugénisme libéral, la marque d'une entreprise intellectuelle originale, décisive et audacieuse parfaitement réussie. Au-delà de la liberté de la recherche, d'éventuels profits de l'industrie biotechnologique, des intérêts légitimes des parents, «des pressions de gouvernements nationaux qui réclament la réussite», ${ }^{18}$ il réaffirme l'importance d'une réflexion philosophique dans la clarification analytique et normative du débat biogénétique. La perspective préventive qu'il inaugure permet de donner un contour relativement précis au débat sur la technique génétique. Elle lui permet notamment de se demander, par anticipation, si nous voulons encore tout simplement continuer à nous comprendre

${ }^{18}$ ANH, p. 33. comme des êtres normatifs ou si nous voulons saper les conditions naturelles de la liberté, de l'égalité et de la responsabilité. De la réponse à cette question dépend l'avenir de la nature humaine. Aussi dispose-t-il qu'en l'absence de raisons contraignantes pour la majorité des sociétaires, il faut interdire les interventions génétiques à cause de ses effets pervers.

Néanmoins, en établissant une version aussi forte du principe de précaution qui, à toutes fins pratiques, interdit totalement la technique génétique à cause de ses conséquences eugéniques, Habermas pèche par excès contre les prémisses d'une éthique postmétaphysique. Car un tel principe ne peut réussir qu'au prix d'un regrettable atavisme métaphysique reposant sur une anthropologie essentialiste de la nature humaine et une surévaluation de l'abîme ${ }^{19}$ séparant le hasard

${ }^{19}$ Cet argument vaut également pour la coupure pertinente qui déterminerait le statut moral de l'embryon. Une telle coupure est difficile à conceptualiser. Habermas lui-même remarque que, quelle que soit la tentative préconisée, il «reste attachée une part d'arbitraire, parce que, ajoute-t-il, la vie, sensible d'abord puis personnelle, se développe depuis ses origines organiques avec une grande continuité» ( $A N H$, p. 53). Si l'on admet une thèse minimale de la continuité, il devient impraticable de moraliser la nature humaine et de fonder une thèse véritablement éthique sur une base naturaliste à partir de la fracture entre le naturel et la fabrication. Cette difficulté aporétique n'est pas seulement conceptuelle, elle est aussi empirique, car «l'expérience nous dégrise» ( $A N H$, p. 43). La Cour suprême du Canada, dans un récent arrêt Harvard College c. Canada (Commission aux brevets), [CSC 76 No. du greffe: 28155 (5 décembre 2002), voir l'article 2 de la Loi sur les brevets, L.R.C. 1985, ch. P-4.] en a fait une laborieuse expérience en essayant de qualifier la «nature» d'une souris modifiée génétiquement. Voir également l'article 2 de la Loi sur les brevets, L.R.C. 1985, ch. P-4. 
de la nature et la décision de fabrication. L'anthropologie sous-jacente à son éthique de l'espèce humaine est paradoxale. Ce paradoxe impliqué par le principe de précaution ne rend pas justice aux réquisits d'une éthique postmétaphysique ne recherchant que les bases neutres d'un consensus minimal. Dans les conditions d'une vie postséculière et pluraliste, l'éthique de l'espèce humaine ne peut retenir que très peu de contenu normatif. Or le contenu que retient le principe habermasien de précaution est massif. Il s'adosse aux prémisses métaphysiques d'arrière-plan du strictement donné, formé par une nature ontologique immuable et unique. Cette posture l'empêche de distinguer nettement les cas qui peuvent échapper à l'instrumentalisation ou l'auto-instrumentalisation de la nature humaine et ceux qui ne le peuvent pas. On peut penser ici à la recherche sur les embryons surnuméraires qui ne sont plus réclamés dans un projet parental. Mis à part le sentiment d'obscénité qu'on peut éprouver à l'idée que des embryons même surnuméraires soient utilisés commé produits pharmaceutiques ou objet de recherche, il n'existe aucun lien direct avec quelque forme d' instrumentalisation de la vie humaine. La recherche sur ces embryons ne présente qu'une relation ténue et très indirecte avec la dignité de la vie humaine.

De plus, il apparait fort peu vraisemblable que la thèse sur laquelle se fonde Habermas, celle des conditions naturelles de croissance, distinguant le hasard de la nature et la décision humaine, le donné et le produit, soit acceptable par tous. Non seulement, elle n'est pas neutre par rapport aux différentes visions du monde mais elle ne prend pas suffisamment en compte la sollicitude, solidarité et la compassion qu'une instrumentalisation régulée peut avoir pour l'image de l'humanité meurtrie et malade. De la sorte, elle sousestime la capacité de la liberté humaine de s'arracher aux déterminations de la nature. 